\title{
Forecasting the Volatility of Nikkei 225 Futures
}

Instituto

Complutense

de Análisis

Económico

\author{
Manabu Asai \\ Faculty of Economics Soka University, Japan
}

\section{Michael McAleer}

Department of Quantitative Finance National Tsing Hua University, Taiwan and Econometric Institute Erasmus School of Economics Erasmus

University Rotterdam, The Netherlands and

Department of Quantitative Economics Complutense University of Madrid, Spain

And Institute of Advanced Sciences Yokohama National University, Japan

\begin{abstract}
For forecasting volatility of futures returns, the paper proposes an indirect method based on the relationship between futures and the underlying asset for the returns and time-varying volatility. For volatility forecasting, the paper considers the stochastic volatility model with asymmetry and long memory, using high frequency data for the underlying asset. Empirical results for Nikkei 225 futures indicate that the adjusted $R 2$ supports the appropriateness of the indirect method, and that the new method based on stochastic volatility models with the asymmetry and long memory outperforms the forecasting model based on the direct method using the pseudo long time series.
\end{abstract}

Keywords Forecasting; Volatility; Futures; Realized Volatility; Realized Kernel; Leverage Effects; Long Memory.

JEL Classification C22, C53, C58, G17

\section{Working Paper no 1707}

UNIVERSIDAD

COMPLUTENSE MADRID

\author{
January, 2017
}




\title{
Forecasting the Volatility of Nikkei 225 Futures*
}

\author{
Manabu Asai \\ Faculty of Economics \\ Soka University, Japan \\ Michael McAleer \\ Department of Quantitative Finance \\ National Tsing Hua University, Taiwan \\ and \\ Econometric Institute \\ Erasmus School of Economics \\ Erasmus University Rotterdam, The Netherlands \\ and \\ Department of Quantitative Economics \\ Complutense University of Madrid, Spain \\ and \\ Institute of Advanced Sciences \\ Yokohama National University, Japan
}

Revised: January 2017

\footnotetext{
${ }^{*}$ The authors are most grateful to Yoshi Baba, Karen Lewis, Robert Webb and an anonymous reviewer for very helpful comments and suggestions. The first author acknowledges the financial support of the Japan Ministry of Education, Culture, Sports, Science and Technology, Japan Society for the Promotion of Science (JSPS Grant Number JP16K03603), and Australian Academy of Science. The second author is most grateful for the financial support of the Australian Research Council, National Science Council, Ministry of Science and Technology (MOST), Taiwan, Japan Society for the Promotion of Science, and Institute of Advanced Sciences, Yokohama National University. Address for correspondence: Faculty of Economics, Soka University, 1-236 Tangi-cho, Hachioji, Tokyo 192-8577, Japan. Email address: m-asai@soka.ac.jp.
} 


\begin{abstract}
For forecasting volatility of futures returns, the paper proposes an indirect method based on the relationship between futures and the underlying asset for the returns and time-varying volatility. For volatility forecasting, the paper considers the stochastic volatility model with asymmetry and long memory, using high frequency data for the underlying asset. Empirical results for Nikkei 225 futures indicate that the adjusted $R^{2}$ supports the appropriateness of the indirect method, and that the new method based on stochastic volatility models with the asymmetry and long memory outperforms the forecasting model based on the direct method using the pseudo long time series.
\end{abstract}

Keywords: Forecasting; Volatility; Futures; Realized Volatility; Realized Kernel; Leverage Effects; Long Memory.

JEL classifications: C22, C53, C58, G17 


\section{Introduction}

It is essential to forecast financial market volatility in both the short and long run. For example, under the Basel II Accord, banks and other authorized deposit-taking institutions need to use short-term volatility forecasts to produce daily Value-at-Risk (VaR) measures, while they use longer term volatility forecasts for option pricing and asset allocation. However, most research has focused on equity markets, foreign exchange markets, and their accompanying options, and studies on modeling and forecasting volatility of returns on futures contracts are limited (see Simon (2002) and Hong, Nohel, and Todd (2015) for the options trading).

One reason for this gap in the literature is that the available sample size before each maturity date is generally insufficient to use time series models, such as autoregressive moving-average (ARMA), autoregressive fractionally integrated moving-average (ARFIMA), and generalized autoregressive conditional heteroskedasticity (GARCH) models. In order to alleviate this problem, the datasets used in Jorion (1995), Martens (2002), Sadorsky (2006), Lai and Sheu (2010), and Lai (2015), among others, are based on the prices of futures contracts closest to maturity, in order to connect small-sized datasets to create a pseudo long time series.

For connected return data for petroleum futures contracts, Sadorsky (2006) compared several statistical models, and found that the asymmetric GARCH model gave the best forecasts. For modeling the joint distribution of spot and futures returns of the S\&P 500 index, Lai and Sheu (2010) and Lai (2015) use realized volatility (RV), which is a direct estimate of latent volatility, without specifying explicit models. Extending the work of Kroner and Sultan (1993), Lai and Sheu (2010) and Lai (2015) developed the RV-based bivariate asymmetric GARCH models (see Barndorff-Nielsen et al. (hereafter BHLS; 2008) and McAleer and Medeiros (2011) for recent developments regarding RV). For connecting the datasets, as the information on time to maturity and overlapping data are discarded, the potential to improve forecasts exists. Unlike Sadorsky 
(2006), Lai and Sheu (2010) and Lai (2015), the paper uses unconnected datasets and considers volatility forecasts as a function of each maturity date.

The paper develops a new approach for forecasting the volatility of futures returns, based on the following relationship between the futures contract and underlying asset. Theoretically, the futures price depends on the price of the underlying asset and the time to maturity. Therefore, the futures volatility may depend on the volatility of the underlying asset and the quadratic function of the time to maturity. If the volatility forecasts of the underlying asset are accurate, it is possible to forecast accurately the volatility of the futures contract. For this reason, the new method also uses the volatility forecasts of the underlying asset.

This paper accommodates two important features, namely the leverage effect and long memory, in order to develop three kinds of volatility forecasting models. One is the SV model, with asymmetry and long memory, using high frequency data. This model is based on the work of Andersen et al. (2003), Andersen, Bollerslev and Meddahi (2005), and Asai, McAleer and Medeiros (2012). Another model is the heterogeneous autoregressive model, with asymmetric effects, which is a variant of Corsi (2009), Martens et al. (2009) and Corsi and Renò (2010). The third model is the fractionally integrated exponential GARCH model of Bollerslev and Mikkelsen (1996). Based on the volatility forecasts of the underlying asset, this new approach is indirect, and is based on the relationships between the volatilities of the spot and futures returns. The new approach also extends previous work, including Jorion (1995), Martens (2002), and Sadorsky (2006), as the new model accommodates long memory.

In order to overcome the small sample problem referred to above, we may consider an alternative approach based on GARCH family models using intraday return series, as investigated by Andersen and Bollerslev (1998). In using intraday returns observed every minute, for example, it is appropriate to accommodate U-shaped intraday periodicity in volatility, as investigated by 
Wood et al. (1985), Harris (1986), and Andersen and Bollerslev (1997), among others. In this case, it is difficult to use daily realized volatility for the differences in frequency. We will not examine this issue further as it moves away from the topic of daily volatility estimates, and is beyond the scope of this paper.

Using the new approach, we can avoid connecting futures data to construct a pseudo long time series. When new futures contracts are launched in the market, we can obtain the volatility forecasts more efficiently from the volatility of the underlying asset, using the relationship between spot and futures volatility. In the empirical analysis, we find that the long range dependence in the connected data often yields a bias in forecasting the futures return volatility, and that the datasets support the use of indirect methods rather than a direct method via the connected datasets.

The paper considers newly launched and illiquid futures markets. First, as our approach is based on the theoretical relationship between futures prices and their underlying assets, it is worth discussing liquidity effects on their markets. Oehmke (2009) develops a model to show that the stock prices of the illiquid segment of the cash index will subsequently align with the index or index futures level, suggesting that arbitrage is slower in more illiquid markets. The recent empirical analysis of Fung, Lau, and Tse (2015) supports the theoretical result for RV in the Hong Kong Hang Seng Index futures. As illiquid markets reduce arbitrage efficiency and weaken the link between futures prices and their underlying stocks, our approach may not work satisfactorily in this situation. We should note that a simple autoregressive model for RV with asymmetric effects, which will be used in the empirical analysis, is expected to show better forecasting performance than existing approaches. Second, we consider a case where a single underlying asset is used for several markets with different maturities. In this case, each market has a different volatility, especially for the newly launched market. Although the existing approaches are unable to provide multiple volatility estimates, our approach is able to do so. We will not extend the issues as they 
are beyond the scope of the paper. In this paper, we use Nikkei 225 futures, which has relatively highly liquid derivatives.

The organization of the remainder of the paper is as follows. Section 2 reviews the volatility estimator of BHLS (2008), and discusses the theoretical relationships between futures and their underlying assets regarding returns and time-varying volatility. Based on the relationship between the volatilities of futures and spot returns, Section 2 proposes a new approach for forecasting the volatility of futures. Section 3 explains three kinds of volatility forecasting models for the underlying asset that are used in the new approach. Section 4 presents the empirical results using high frequency Nikkei 225 futures data and the underlying Nikkei Stock Average Index (Nikkei 225). Section 5 gives some concluding remarks.

\section{A New Approach for Forecasting Volatility of Futures Returns}

\section{$2.1 \quad$ Realized Kernel}

Let $Y_{\tau}$ be the log-price of a financial asset at time $\tau$ on day $t$, where $\tau \in[t-1, t]$. We assume that $Y_{\tau}$ follows the Brownian semimartingale plus jump (BMSJ) process:

$$
Y_{\tau}=\int_{t-1}^{\tau} a_{u} d u+\int_{t-1}^{\tau} \sigma_{u} d W_{u}+J_{\tau}
$$

where $J_{\tau}=\sum_{i=1}^{N_{\tau}} C_{i}$ is a finite activity jump process, $N_{\tau}$ denotes the number of finite jumps in the interval $[t-1, \tau]$, with $N_{\tau}<\infty$ for any $t, a_{u}$ is the drift term, $\sigma_{u}$ denotes the volatility, and $W_{u}$ is a standard Brownian motion process. For any deterministic partition $t-1=\tau_{0}<\tau_{1}<\cdots<\tau_{n}=t$, the quadratic variation $(\mathrm{QV})$ at day $t$ is defined by:

$$
[Y]_{t}=\operatorname{plim} \sum_{j=1}^{\tau_{j} \leq t}\left(Y_{\tau_{j+1}}-Y_{\tau_{j}}\right)^{2}=\int_{t-1}^{t} \sigma_{u}^{2} d u+\sum_{i=1}^{N_{t}} C_{i}^{2}
$$

where $\int_{t-1}^{t} \sigma_{u}^{2} d u$ is the integrated variance, and it is assumed that $\sup _{j}\left\{\tau_{j+1}-\tau_{j}\right\} \rightarrow 0$ as $n \rightarrow \infty$. 
Define the noisy observation of $Y_{\tau_{j}}$ as $X_{\tau_{j}}=Y_{\tau_{j}}+U_{\tau_{j}}$, where $U_{\tau_{j}}$ denotes the noise with mean zero and constant variance. The noise can be caused by, for example, liquidity effects, bid/ask bounce and mis-recording. We estimate daily QV from the observations $X_{\tau_{0}}, \ldots, X_{\tau_{n}}$. A simple approach is to use realized variance (RV), which is defined by $\sum_{j=1}^{n} x_{j}^{2}$, where $x_{j}$ is the $j$ th high frequency return calculated over the interval $\left[\tau_{j-1}, \tau_{j}\right]$, defined by $x_{j}=X_{\tau_{j}}-X_{\tau_{j-1}}$. Unfortunately, $\mathrm{RV}$ is inconsistent in the presence of microstructure noise.

There are two popular approaches for estimating QV. One is the realized kernel (RK) estimator of BHLS (2008), while the other is the two time scale realized volatility (TSRV) estimator of Zhang et al. (2005) and Aït-Sahalia et al. (2011). These estimators are robust to microstructure noise. Among them, we use the RK estimator, since it is the only estimator which is robust to jumps. For estimating daily QV, BHLS (2008) developed the non-negative estimator which takes the following form:

$$
K_{t}(X)=\sum_{h=-H}^{H} k\left(\frac{h}{H+1}\right) \gamma_{k}, \quad \gamma_{k}=\sum_{j=|h|+1}^{n} x_{j} x_{j-|h|},
$$

where $k(x)$ is a kernel weight function. Following BHLS (2009), we use the Parzen kernel function:

$$
k(x)= \begin{cases}1-6 x^{2}+6 x^{3} & 0 \leq x \leq 1 / 2 \\ 2(1-x)^{3} & 1 / 2 \leq x \leq 1 \\ 0 & x>1\end{cases}
$$

The estimator takes the same form as the standard heteroskedasticity and autocorrelated (HAC) covariance matrix estimator (e.g. Newey and West (1987) and Andrews (1991)), but $K_{t}(X)$ is not normalized by the sample size. As shown in BHLS (2008), $K_{t}(X)$ is a consistent estimator of $[Y]_{t}$ (see BHLS $(2008,2009)$ for further details on the selection of an appropriate value of $H$ ). By construction, the estimator is called RK. The estimate of volatility is obtained by the square root of the RK estimate. 


\subsection{The Relationship between Spot and Futures Volatility}

Let $R_{t}$ be the logarithmic return of the underlying asset on day $t$, and let $f_{t}$ be the futures return with the time to maturity, $T$. The relationship between $f_{t}$ and $R_{t}$ is given by $f_{t}=R_{t}-(\delta-r) T$, where $r$ is the risk-free rate, and $\delta$ is the expected dividend yield. The relationship leads to a regression model:

$$
f_{t}=\alpha_{r}+\beta_{r} R_{t}+\gamma_{r}(-T)+\varepsilon_{t},
$$

with the restrictions $\alpha_{r}=0$ and $\beta_{r}=1$, where $\varepsilon_{t}$ is the disturbance term. The estimate of $\gamma_{r}$ can be interpreted as the estimate of $(\delta-r)$.

Turning to the time-varying volatility, let $R K_{t}$ and $R K_{t}^{f}$ be the realized kernel estimates of the variance of the spot and futures returns, respectively. By the above relationships, we have $\sum_{j=1}^{n} f_{j t}^{2}=\sum_{j=1}^{n} R_{j t}^{2}+2(\delta-r) T \sum_{j=1} R_{j t}+n(\delta-r) T^{2}$, where $f_{j t}\left(R_{i t}\right)$ is the $i$ th futures (spot)

return at day $t$. By adding cross-products, $f_{j t} f_{j-|h|, t}$ and $R_{j t} R_{j-|h|, t}$, to this equation, as in (1), we obtain the relationship that $R K_{t}^{f}$ is affected by, not only $R K_{t}$, but also by the time to maturity and its square. Hence, we consider the regression model:

$$
R K_{t}^{f}=\alpha_{v}+\beta_{v} R K_{t}+\gamma_{v 1} T+\gamma_{v 2} T^{2}+v_{t}
$$

with the restrictions $\alpha_{v}=0$ and $\beta_{v}=1$, where $v_{t}$ is the disturbance term.

We estimate parameters of equations (2) and (3), using the OLS and HAC covariance matrix estimators. We construct the robust $\chi^{2}$ test statistic for testing the restrictions using the HAC covariance matrix estimator.

\subsection{Forecasting Volatility of Futures Returns}

There are alternative techniques for forecasting spot return volatilities, as explained in the introduction. It is not easy to develop forecasting models for the volatility of the futures return as 
the sample size is not large enough for estimation, except for simple autoregressive (AR) models. The new approach for forecasting the volatility of futures return is based on the spot-futures relationship for the time-varying variance (3), and the forecasts of spot return volatility.

Corresponding to day $t(t=1,2, \ldots)$ for the underlying asset, we denote $t=n_{0}$ as the previous day to when trading of the futures starts, and denote the maturity date as $t=n_{0}+n_{f}$. Hence, futures are traded for $n_{f}$ days. The new approach estimates the initial relationship, using estimated time-varying variances of spot and futures returns for $q$ days, $R K_{n_{0}+h}$ and $R K_{n_{0}+h}^{f}$ $(h=1,2, \ldots, q)$, respectively, starting from $q=5$ in the empirical analysis given below.

On day $t=n_{0}+q$, the one-step-ahead forecast of the volatility of futures is obtained using the following two steps:

Step 1: For the underlying asset, obtain the one-step-ahead forecast, $\widehat{R K}_{n_{0}+1}$, using the estimated time-varying variances, $R K_{t}\left(t=1, \ldots, n_{0}\right)$. Fixing the sample size as $n_{0}$, update $R K_{n_{0}+1}$ and obtain $\widehat{R K}_{n_{0}+2}$. Repeat the process $q+1$ times in order to obtain the series of one-step ahead forecasts, $\widehat{R K}_{n_{0}+h}(h=1,2, \ldots, q+1)$.

Step 2: For the futures return, obtain the estimates of time-varying variances, $R K_{n_{0}+h}^{f}(h=$ $1,2, \ldots, q)$, and estimate the parameters of the regression model:

$$
R K_{t}^{f}=\alpha_{v}+\beta_{v} \widehat{R K}_{t}+\gamma_{v 1} T+\gamma_{v 2} T^{2}+v_{t}, \quad\left(t=n_{0}+1, n_{0}+2, \ldots, n_{0}+q\right)
$$

using OLS. Setting $\widehat{R K}_{n_{0}+q+1}$ in the right-hand-side gives the predicted value, $\widehat{R K}_{n_{0}+q+1}^{f}$.

The proposed approach can easily be extended to obtain 5 and 10 step-ahead forecasts. After obtaining the new estimates of the time-varying variances, set $q=q+1$, and repeat Steps 1 and 2 until $q=n_{f}-1$, which corresponds to the prior day to the maturity date. The volatility forecasts of futures returns are obtained by taking the square root of $\widehat{R K}_{n_{0}+h}^{f}\left(h=q+1, \ldots, n_{f}\right)$. 
As the process starts from $q=5$, we cannot use the method in the first week of newly launched markets. For the first week, we may use simple methods, such as a moving-average of past $R K^{f} \mathrm{~s}$ for predicting volatility.

\section{Volatility Forecasting Models for the Underlying Asset}

Consider the following stochastic volatility model with asymmetry and long memory (ALSV):

$$
\begin{aligned}
& R_{t}=V_{t}^{1 / 2} z_{t}, \quad z_{t} \sim i i d(0,1), \\
& (1-\phi L)(1-L)^{d}\left(\ln V_{t}-\mu\right)=\xi_{t}, \\
& \xi_{t}=\lambda_{1} z_{t-1}+\lambda_{2}\left(z_{t-1}^{2}-1\right)+\eta_{t}, \quad \eta_{t} \sim i i d\left(0, \sigma_{\eta}^{2}\right),
\end{aligned}
$$

where $V_{t}$ is the stochastic variance, $L$ is the lag operator, $z_{t}$ and $\eta_{t}$ are independent processes, and $d, \phi, \lambda_{1}, \lambda_{2}$ and $\sigma_{\eta}$ are scalar parameters. $z_{t}$ may have a heavy-tailed distribution, but it is assumed that $E\left(z_{t}^{4}\right)$ is finite. As $\xi_{t}$ has mean zero and finite variance, by construction, $\ln V_{t}$ follows the $\operatorname{ARFIMA}(1, d, 0)$ process, with the parameter of fractional difference, $d$. It is also possible to consider an ARFIMA(5,d,0) model as in Andersen et al. (2003) and Andersen, Bollerslev and Meddahi (2005). For stationarity and invertibility, the process needs to assume that $|\phi|<1$ and $|d|<1 / 2$. If $\lambda_{1}<0$ and $\lambda_{2} \geq 0, z_{t}$ and $\xi_{t+1}$ are negatively correlated, yielding leverage effects.

Furthermore, $\lambda_{1} z_{t-1}+\lambda_{2}\left(z_{t-1}^{2}-1\right)$ can be considered as the second-order approximation of the Hermite polynomials. Chen and Ghysels (2010) and Asai, McAleer and Medeiros (2012) provide alternative specifications.

Although $V_{t}$ is the latent variable in the ALSV model, it can be replaced by the realized kernel estimate. As discussed in Barndorff-Nielsen and Shephard (2002), the 'RV error', which is the difference between the true and realized volatility, causes bias in estimating the parameters of stochastic volatility models. The simulation experiments of Asai, McAleer and Medeiros (2012b) indicate that the estimation bias is severe, but that the effects of the RV error are negligible 
in forecasting volatilities. For this reason, the above model works by setting $V_{t}=R K_{t}$ and $z_{t}=R_{t} / \sqrt{R K_{t}}$ (see also Andersen et al. (2003) and Andersen, Bollerslev and Meddahi (2005), and Bollerslev et al. (2009)). The following two-step approach is used for estimating the ALSV model: The first step estimates the long memory parameter, $d$, by the local Whittle (LW) estimator of Shimotsu and Phillips (2006); the second step estimates the remaining parameter using OLS.

For purposes of comparison, the heterogeneous autoregressive (HAR) model of Corsi (2009) and the fractionally-integrated exponential generalized ARCH (FIEGARCH) model of Bollerslev and Mikkelsen (1996) are used. Following Corsi (2009), denote the average of $V_{t}$ for the past $h$ period as $(V)_{t-1: t-h}=h^{-1} \sum_{i=1}^{h} V_{t-i}$. Consider the asymmetric HAR (HAR-A) model:

$$
R K_{t}=\alpha_{c}+\beta_{d} R K_{t-1}+\beta_{w}(R K)_{t-1: t-5}+\beta_{m}(R K)_{t-1: t-22}+\lambda_{1} R_{t-1}+\lambda_{2} R_{t-1}^{2}+e_{t},
$$

where $e_{t}$ is the disturbance term. Note that $(R K)_{t-1: t-5}$ and $(R K)_{t-1: t-22}$ are the weekly and monthly averages, respectively. The HAR model can also be considered as the AR(22) model with restrictions, and hence it is expected to capture longer effects than the conventional AR(1) and/or AR(5) models. The estimation of the HAR-A model can be conducted by OLS. It is also possible to use the indicator function and thresholds to describe asymmetric effects, as in Martens et al. (2009) and Corsi and Renò (2010).

The FIEGARCH model used in the paper is obtained from the ALSV model (5) by removing the disturbance term, $\eta_{t}$. By the elemination, $\xi_{t}$ is determined by the information set up to $t-1$, giving the deterministic value of $V_{t}$, which means the reduced model belongs to the $\mathrm{ARCH}$ family. Instead of $\left(z_{t-1}^{2}-1\right)$, which is the only difference, Bollerslev and Mikkelsen (1996) work with $\left(\left|z_{t-1}\right|-E\left|z_{t-1}\right|\right)$, as in Nelson (1991). The FIEGARCH model can be estimated by the quasi-maximum likelihood method.

All three models accommodate long memory and asymmetric effects in their volatility structures. Compared with RK-ALSV, the RK-HAR-A model approximates the long memory, and the 
FIEGARCH model is less flexible as it omits the disturbance, $\eta_{t}$. Hence, the RK-ALSV model is expected to fit the data better than do the other two models. We should note that there is no guarantee that the model which gives the best fit produces the best forecasting performance.

As discussed in the previous section, the RK-ALSV, RK-HAR-A, and FIEGARCH models are used for Step 1 in forecasting the time-varying variances of futures returns. The forecasts of volatility are obtained by taking the square root of $\widehat{R K}_{n_{0}+h}\left(h=1, \ldots, n_{f}\right)$.

\section{Empirical Analysis}

\subsection{Data and Preliminary Results}

The data for the empirical analysis in the paper consists of high frequency Nikkei 225 futures contracts and their underlying Nikkei 225 index for the period January 4, 2012 to October 31, 2014. Nikkei 225 futures are traded on the Osaka Exchange (OSE), Japan, and the contracts months are March, June, September and December. The paper focuses on the twelve kinds of contracts where the maturity dates are included in the years 2012 to 2014 . Trading hours consist of day and night sessions, namely 9:00am-3:15pm, 4:30pm-3:00am. Data for the day session are used for simplicity. The open-close returns and RKs are calculated using 1-minuet data, which are provided by the OSE.

The underlying equity of the futures is the Nikkei 225, which is comprised of 225 stocks listed on the Tokyo Stock Exchange First Section. Similarly, the returns and RKs are calculated using the 15-second data, provided by the Index Business Office, Nikkei Inc. Figure 1 shows the daily return and volatility for Nikkei 225, with large fluctuations starting from May 23, 2013 until the middle of August, 2013. We use the open-close return instead of the close-close return, following Hansen Huang, and Shek (2012).

Table 1 presents the descriptive statistics for Nikkei 225 futures. Corresponding to the above

period, $f_{t}$ and $R K_{t}^{f}$ with contract months which are June, September and December, 2013 has a 
higher sample standard deviation than the others. As the sample size is 148 at most, it is hard to apply conventional ARMA and GARCH models to obtain forecasts for most of the sample period.

Table 2 shows the OLS estimates for regression (2), which is based on the theoretical relationship between the spot and futures returns. Table 2 indicates that all estimated coefficients are significant at the $1 \%$ level. Although the estimates of the constant term $\left(\alpha_{r}\right)$ are close to zero and those of the coefficient of $R_{t}\left(\beta_{r}\right)$ are close to one, the null hypothesis, $H_{0}: \alpha_{r}=0, \beta_{r}=1$, is rejected in all cases, except for the contract month of June, 2012. All values of adjusted $R^{2}$ reported in Table 2 are higher than 0.84 .

Table 3 reports the OLS estimates for regression (3), which is based on the theoretical relationship between the spot and futures time-varying volatility. Table 3 indicates that the constant term $\left(\alpha_{v}\right)$ and the estimated coefficient of $R K_{t}\left(\beta_{v}\right)$ are significant. The estimates of $\alpha_{v}$ are far from zero in most cases, while the estimates of $\beta_{r}$ are close to one. The null hypothesis, $H_{0}: \alpha_{v}=0$, $\beta_{v}=1$, is rejected in all cases. The robust $\chi^{2}$ test for the null hypothesis, $H_{0}: \gamma_{v 1}=\gamma_{v 2}=0$, is

also rejected, although the results are not reported in Table 3 . All of the values of adjusted $R^{2}$ reported in Table 3 are higher than 0.91, implying the strong relationships between the spot and futures volatilities.

The small sample size of the futures contracts indicates that it is difficult to work with conventional time series models in forecasting time-varying variances. The preliminary results suggest that, if the forecasts of the volatility of the spot equity returns are accurate, reliable forecasts of futures volatility can be obtained via the relationship between the spot and futures volatilities.

\subsection{Volatility Forecasts}

This subsection compares the accuracy of the one-step-ahead forecasts of futures volatility via the proposed approach based on the three volatility forecasting models of the underlying Nikkei 225 
returns, namely the RK-ALSV, RK-HAR-A, and FIEGARCH models. For purposes of comparison, the root mean squared forecast error (RMSFE) and the Diebold and Mariano (1995) test are used. Diebold and Mariano (1995) develop a test of equal forecast accuracy between two sets of forecasts, using the mean squared error. The test of the null hypothesis of equal forecast accuracy is based on $E\left(d_{t}\right)=0$, where $d_{t}=e_{1 t}^{2}-e_{2 t}^{2}$, and $e_{1 t}$ and $e_{2 t}$ are forecast errors from models 1 and 2, respectively. The Diebold and Mariano (1995) test statistic is given by $Z=\bar{d} / \sqrt{\hat{V}(\bar{d})}$, where $\bar{d}=n^{-1} \sum_{t=1}^{n} d_{t}$, and $\hat{V}(\bar{d})$ is given by the HAC-type estimator. Under the null hypothesis, $Z$ has an asymptotic normal distribution.

One-step-ahead forecasts via the RK-ALSV, RK-HAR-A, and FIEGARCH models for volatilities of the underlying Nikkei 225 returns are obtained by the rolling window method, fixing $n_{0}=250$, and the forecasting period is from March 1, 2013 to October 31, 2014. Table 4(a) shows that the RK-ALSV model has the smallest RMSFE, while the FIEGARCH model has the largest RMSFE. Table 4(b) shows the results of the Diebold and Mariano (1995) test. The null hypothesis that there is no difference in the forecasts between two models is rejected at $5 \%$ significance level for all three combinations, implying that there are significant differences in the forecasts of the three models at the $5 \%$ level.

Table 5 reports the RMSFE for one-step-ahead forecasts for the volatility of Nikkei 225 futures, based on the approach proposed in Section 2, for the futures of 6 kinds of contract months. In addition to the proposed approach, the simple AR(1) model with asymmetric effects (AR(1)-A):

$$
R K_{t}^{f}=\alpha_{0}+\beta_{0} R K_{t-1}^{f}+\lambda_{1} f_{t-1}+\lambda_{2} f_{t-1}^{2}+e_{t},
$$

is used as the benchmark model. The $\mathrm{AR}(1)$-A model based on the past values of $R K_{t}^{f}$ and $f_{t}$ outperforms the new indirect method based on the FIEGARCH model in five of six cases. The indirect approach based on RK-ALSV has the smallest RMSFE in all six cases. The results of the Diebold and Mariano test indicate that there are significant differences between the forecasts of 
the RK-ALSV model and the second best model in four of the six cases.

\subsection{Analysis on Connected Futures Data}

In this subsection, we compare the RMSFE of the indirect method with the direct method, using the connected futures data, as in Jorion (1995), Martens (2002), Sadorsky (2006), Lai and Sheu (2010), and Lai (2015). For the connected futures data, we use the realized kernel estimates of daily volatility, $R K_{t}^{f}$, to estimate the HAR-A and ALSV models, and to obtain the one-step-ahead forecasts. We may classify the $\mathrm{RK}^{f}$-HAR-A and RK ${ }^{f}$-ALSV models, and the AR(1)-A model in the previous section, as direct methods for obtaining forecasts of futures return volatility.

Table 6 presents the RMSFE for these six models. Among the direct methods, the RK ${ }^{f}-\mathrm{HAR}$ A, $\mathrm{RK}^{f}$-ALSV and AR(1)-A models yield similar results. For all models, the indirect method based on the RK-ALSV model has the smallest RMSFEs except for the last period. For the last period, the difference in RMSFEs between the best model and RK-ALSV are negligible. The empirical results imply that long range dependence plays an important role in forecasting volatility. For the connected data, we may consider the dataset before the previous maturity date as a proxy for the true data. If the proxy is close to the true data, we will have accurate forecasts using the $\mathrm{RK}^{f}$-HAR-A and $\mathrm{RK}^{f}$-ALSV models. On the other hand, if the proxy is not close to the true, the long range dependence in the proxy data will give misleading forecasts. For this reason, the simple AR(1)-A model often outperforms the $\mathrm{RK}^{f}$-HAR-A and $\mathrm{RK}^{f}$-ALSV models in Table 6. For the connected data, the indirect method based on the RK-ALSV model tends to produce better forecasts than do the direct methods.

The empirical results imply the existence of long-range dependence in the futures return volatility. For the disconnected data, the spot return volatility is known to have long memory properties, while the empirical results for the indirect methods indicate strong relationships between the spot and futures volatilities. Regarding the connected data, the direct forecasts via long memory mod- 
els show the differences from those of the simple AR(1)-A model. However, the long memory models are often inferior to the AR(1)-A model, as the detected long memory in the connected data often causes a bias in forecasting. The empirical analysis here compares the accuracy of the direct method with biased long memory, the direct one without long memory, and the indirect method with accurate long memory. The results show that the last method above produces the best forecasting performance for the Nikkei 225 futures volatility.

\subsection{Utility-Based Measure}

Consider a risk averse investor (hedger), who wishes to use futures to reduce the price risk of his or her spot portfolio, with quadratic utility. Denote the hedged portfolio return from day $t$ to $t+1$ as $r_{p, t+1}=r_{t+1}-\theta_{t} f_{t+1}$, where $\theta_{t}$ is the hedge ratio determined by the information up to day $t$. The optimal hedge ratio is given by:

$$
\theta_{t}^{*}=\rho_{t} \sqrt{\frac{\operatorname{Var}_{t}\left(r_{t+1}\right)}{\operatorname{Var}_{t}\left(f_{t+1}\right)}},
$$

where $\operatorname{Var}_{t}\left(x_{t+1}\right)$ and $\rho_{t}$ are the conditional variance of $x_{t+1}$, and the conditional correlation between $r_{t+1}$ and $f_{t+1}$, respectively. We use the one-step-ahead forecasts of volatilities of spot and futures returns as $\operatorname{Var}_{t}\left(r_{t+1}\right) / \operatorname{Var}_{t}\left(f_{t+1}\right)$, calculated by the indirect methods based on the FIEGARCH, RK-HAR-A, and RK-ALSV models. We also use the futures volatility forecasts based on the AR(1)-A model, paired with the spot volatility forecasts via FIEGARCH, for constructing the baseline portfolio. For the value of $\rho_{t}$, we calculated the correlation coefficient of $r_{s}$ and $f_{s}$ $(s=1, \ldots, t)$, as an ad hoc approach. We should note that, for obtaining the values of the optimal portfolio, we are unable to use the multivariate GARCH models and their extensions, as in Baillie and Myers (1991), Kroner and Sultan (1993), Lai and Sheu (2010), and Lai (2015), among others, the available sample size before the maturity date is insufficient for using such models.

Fleming et al. (2001, 2003) suggested a utility-based approach to measure the value of performance gains associated with the volatility forecasts. The daily utility generated by this portfolio 
is given by:

$$
U\left(r_{p, t}\right)=W_{0}\left(\left(1+r_{f}+r_{p, t}\right)-\frac{\psi}{2(1+\psi)}\left(1+r_{f}+r_{p, t}\right)^{2}\right)
$$

where $\psi$ is the investor's relative risk aversion, and $r_{f}$ denotes the risk-free interest rate. Note that the quadratic utility function can be considered as a second-order approximation to the investor's true utility function.

Let $r_{p_{1}, t}$ and $r_{p_{2}, t}$ denote the returns using two different portfolios. Following Fleming et al. (2001, 2003), we measure the incremental value of using the second portfolio instead of the first, by obtaining a constant $\Delta$ which satisfies:

$$
\sum_{t=n_{0}+q+1}^{n^{f}} U\left(r_{p_{2}, t}\right)=\sum_{t=n_{0}+q+1}^{n^{f}} U\left(r_{p_{1}, t}+\Delta\right) .
$$

This constant indicates the maximum return that the investor would be willing to sacrifice each day in order to capture the performance gains by switching from the first portfolio to the second. As in Fleming et al. $(2001,2003)$, we set $r_{f}=0.06$, and report the value of $\Delta$ as an annualized basis point fee, with respect to two kinds of relative risk aversion level, namely $\psi=1$ and $\psi=10$.

Table 7 compares 4 kinds of hedge portfolios. The baseline model uses the AR(1)-A model for forecasting futures volatility, while the other three models use the indirect methods based on the FIEGARCH, RK-HAR-A, and RK-ALSV models. For each model, Table 7 presents the mean next-day portfolio return $(\mu)$, volatility $(\sigma)$, and the basis point fees $\left(\Delta_{\psi}\right)$ which an investor would pay to switch from the static portfolio for relative risk aversion, $\psi$. Table 7 indicates that the indirect method based on the RK-ALSV model outperforms the baseline model in nine of twelve cases. Compared with the other models, the RK-ALSV model gives better and more stable results. 


\section{Conclusions}

As daily observations for one maturity date in a futures market are insufficient to estimate long memory volatility models, samples of different maturity months are combined to create pseudo time series data for estimating and forecasting the volatility of futures returns. As the approach discards the information on time to maturity and overlapping observations, there is potential to improve the forecasts by accommodating these effects. For this purpose, the paper develops an indirect approach based on the relationship between spot and futures volatilities. The empirical results for the volatility of Nikkei 225 futures indicate that the new indirect method based on the RK-ALSV model outperforms the direct forecasting models that use connected datasets.

Extensions of the new approach are possible. First, we can improve the volatility forecasting model for the underlying asset by accommodating the jump component, as in Andersen et al. (2007) and Bollerslev et al. (2009), and by including trading volumes, as in Tauchen and Pitts (1983) and Fleming, Kirby, and Ostdiek (2006), among others. Second, we can improve the relationship between the spot and futures volatilities. Third, we can develop better approaches to construct the hedge portfolio. Fourth, the market of Nikkei 225 futures has relatively high liquidity from the beginning date to its maturity. As discussed in the Introduction, we can investigate the issues of newly launched and illiquid markets. Such tasks await future research. 


\section{Refferences}

Aït-Sahalia, Y., P.A. Mykland, and L. Zhang (2011), "Ultra High Frequency Volatility Estimation with Dependent Microstructure Noise", Journal of Econometrics, 160, 160-175.

Andersen, T. G., and T. Bollerslev (1997), "Intraday Periodicity and Volatility Persistence in Financial Markets", Journal of Empirical Finance, 4, 115-158.

Andersen, T. G., and T. Bollerslev (1998), "Answering the Skeptics: Yes, Standard Volatility Models do Provide Accurate Forecasts", International Economic Review, 39, 885-905.

Andersen, T.G., T. Bollerslev, and F.X. Diebold (2007), "Roughing It Up: Including Jump Components in the Measurement, Modeling and Forecasting of Return Volatility", Review of Economics and Statistics, 89, 701-720.

Andersen, T. G., T. Bollerslev, F. X. Diebold, and P. Labys (2003), "Modeling and Forecasting Realized Volatility", Econometrica, 71, 529-26.

Andersen, T. G., T. Bollerslev, and N. Meddahi (2005), "Correcting the Errors: A Note on Volatility Forecast Evaluation based on High-Frequency Data and Realized Volatilities", Econometrica, 73, $279-96$.

Andrews, D.W.K. (1991), "Heteroskedasticity and Autocorrelation Consistent Covariance Matrix Estimation", Econometrica, 59, 817-858.

Asai, M., M. McAleer and M. Medeiros (2012), "Asymmetry and Long Memory in Volatility Modeling", Journal of Financial Econometrics, 10, 495-512.

Baillie, R. T., and R. J. Myers (1991), "Bivariate GARCH estimation of the optimal commodity futures hedge", Journal of Applied Econometrics, 6, 109-124.

Barndorff-Nielsen, O.E., P.R. Hansen, A. Lunde, and N. Shephard (2008), "Designing Realised Kernels to Measure the Ex-Post Variation of Equity Prices in the Presence of Noise", Econometrica, 76, $1481-1536$.

Barndorff-Nielsen, O.E., P.R. Hansen, A. Lunde, and N. Shephard (2009), "Realised Kernels in Practice: Trades and Quotes", Econometrics Journal, 12, C1-C32.

Barndorff-Nielsen, O.E., and N. Shephard (2002), "Econometric Analysis of Realized Volatility and Its Use in Estimating Stochastic Volatility Models", Journal of the Royal Statistical Society, Series B, 64, 253-280.

Bollerslev, T., U. Kretschmer, C. Pigorsch, and G. Tauchen (2009), "A Discrete-Time Model for Daily S\&P500 Returns and Realized Variations: Jumps and Leverage Effects", Journal of Econometrics, 150, 151-166.

Bollerslev, T. and H.O. Mikkelsen (1996), "Modeling and Pricing Long-Memory in Stock Market Volatility", Journal of Econometrics, 73, 151-184.

Chen, X., and E. Ghysels (2010), "News - Good or Bad - and Its Impact on Volatility Predictions over Multiple Horizons", Review of Financial Studies, 24, 46-81.

Corsi, F. (2009), "A Simple Approximate Long-Memory Model of Realized Volatility", Journal of Financial Econometrics, 7, 174-196.

Corsi, F. and R. Renò (2010), "HAR Volatility Modelling with Heterogeneous Leverage and Jumps", Unpublished Paper, Università di Siena.

Diebold, F. and R. Mariano (1995), "Comparing Predictive Accuracy", Journal of Business \& Economic Statistics, 13, 253-263. 
Fleming, J., C. Kirby and B. Ostdiek (2001), "The Economic Value of Volatility Timing", Journal of Finance, 56, 329-352.

Fleming, J., C. Kirby and B. Ostdiek (2003), "The Economic Value of Volatility Timing Using "Realized" Volatility", Journal of Financial Economics, 67, 473-509.

Fleming, J., C. Kirby, and B. Ostdiek (2006), "Stochastic Volatility, Trading Volume, and the Daily Flow of Information", Journal of Business, 79, 1551-1590.

Fung, J.K.W., F. Lau, and Y. Tse (2015), "The Impact of Sampling Frequency on Intraday Correlation and Lead 豊 ag Relationships Between Index Futures and Individual Stocks", Journal of Futures Markets, 35, 939-952.

Hansen, P.R., Z. Huang, and H.H. Shek (2012), "Realized GARCH: A Complete Model of Returns and Realized Measures of Volatility", Journal of Applied Econometrics, 27, 877-906.

Harris, L. (1986), "A Transaction Data Study of Weekly and Intradaily Patterns in Stock Returns", Journal of Financial Economics, 16, 99-117.

Hong, L., T. Nohel, and S. Todd (2015), "Forecasting Volatility in the Presence of Limits to Arbitrage", Journal of Futures Markets, 35, 987-1002.

Jorion, P. (1995), "Predicting Volatility in the Foreign Exchange Market", Journal of Finance, 50, 507528.

Kroner, K. F., and J. Sultan (1993), "Time-Varying Distributions and Dynamic Hedging with Foreign Currency Futures", Journal of Financial and Quantitative Analysis, 28, 535-551.

Lai, Y.-S. (2015), "Hedge Ratio Prediction with Noisy and Asynchronous High-Frequency Data", to appear in Journal of Futures Markets.

Lai, Y.-S., and H.-J. Sheu (2010), "The Incremental Value of a Futures Hedge using Realized Volatility", Journal of Futures Markets, 30, 874-896.

Martens, M. (2002), "Measuring and Forecasting S\&P 500 Index-Futures Volatility Using High-Frequency Data", Journal of Futures Markets, 22, 497-518.

Martens, M., D. van Dijk, and M. de Pooter (2009), "Forecasting S\&P 500 Volatility: Long Memory, Level Sifts, Leverage Effects, Day-of-the-Week Seasonality, and Macroeconomic Announcements", International Journal of Forecasting, 25, 282-303.

McAleer, M. and M. C. Medeiros (2011), "Forecasting Realized Volatility With Linear And Nonlinear Univariate Models", Journal of Economic Surveys, 25, 6-18.

Nelson, D.B. (1991), "Conditional Heteroskedasticity in Asset Returns: A New Approach", Econometrica, 59, 347-370.

Newey, W.K., and K.D. West (1987), "A Simple Positive Semi-Definite, Heteroskedasticity and Autocorrelation Consistent Covariance Matrix", Econometrica, 55, 703-708.

Oehmke, M. (2009), "Gradual Arbitrage", Working paper, Columbia University.

Sadorsky, P. (2006), "Modeling and Forecasting Petroleum Futures Volatility", Energy Economics, 28, $467-488$.

Shimotsu, K. and P.C.B Phillips (2006), "Local Whittle Estimation of Fractional Integration and Some of Its Variants", Journal of Econometrics, 130, 209-233.

Simon, D.P. (2002), "Implied Volatility Forecasts in the Grains Complex", Journal of Futures Markets, 22, 959-981.

Tauchen, G. E., and M. Pitts (1983), "The Price Variability-Volume Relationship on Speculative Markets", Econometrica, 51, 485-505. 
Wood, R.A., Mclnish, T.H., and J.K. Ord (1985), "An Investigation of Transaction Data for NYSE Stocks", Journal of Finance, 25, 723-739.

Zhang, L., P.A. Mykland, and Y. Aït-Sahalia (2005), "A Tale of Two Time Scales: Determining Integrated Volatility with Noisy High-Frequency Data", Journal of American Statistical Association, 100, 1394411. 
Figure 1: Daily Return and Volatility for Nikkei 225

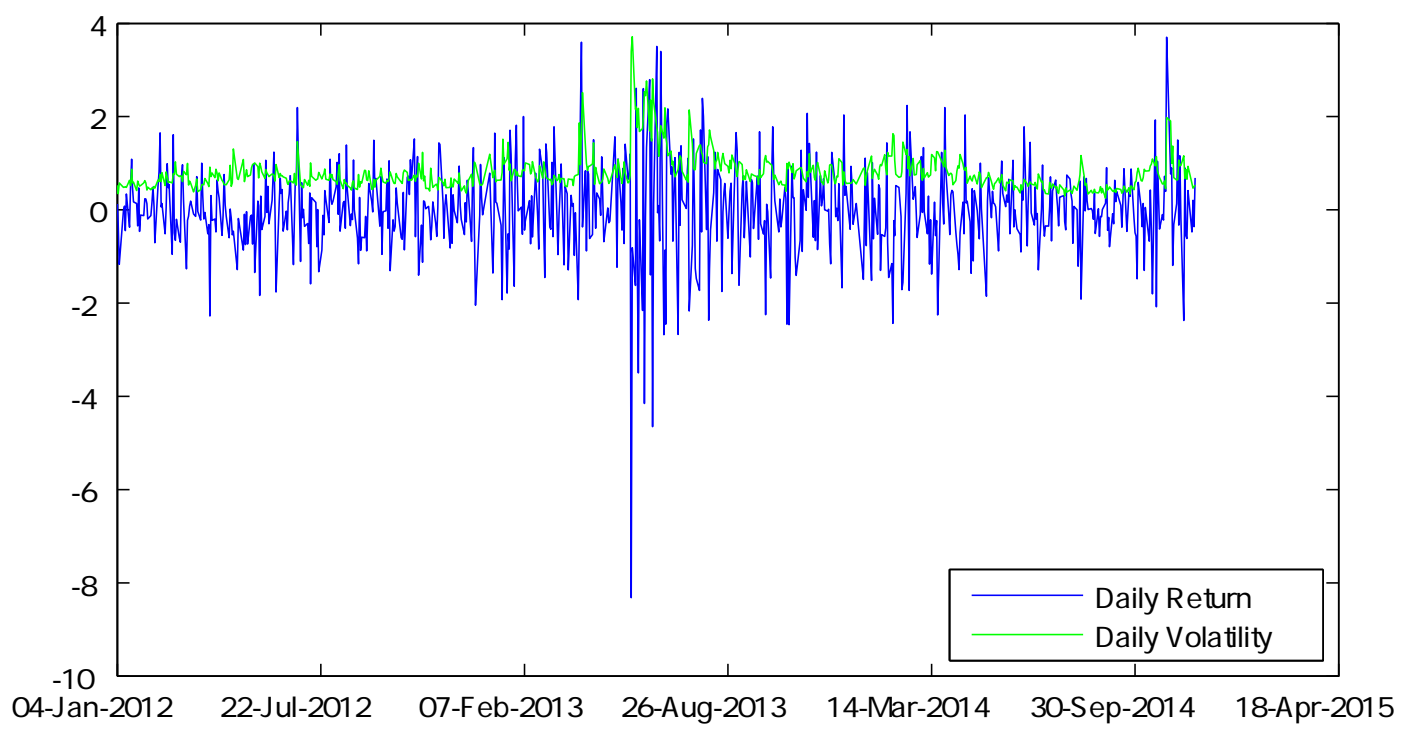

Note: Open-close returns are used, while the volatilities are calculated by taking the square root of RK in equation (1). 
Table 1: Descriptive Statistics for Nikkei 225 Futures

\begin{tabular}{c|rrrrr}
\hline \hline Data & $n^{f}$ & Average & Std. Dev. & Skewness & Kurtosis \\
\hline$f_{t}^{201203}$ & 46 & 0.0718 & 0.6528 & 0.7039 & 3.7455 \\
$f_{t}^{201206}$ & 107 & -0.0502 & 0.6678 & 0.0792 & 4.6563 \\
$f_{t}^{201209}$ & 95 & -0.0234 & 0.7337 & 0.0029 & 3.2024 \\
$f_{t}^{201212}$ & 115 & -0.0180 & 0.6762 & 0.1164 & 2.6953 \\
$f_{t}^{201303}$ & 105 & 0.0332 & 0.7961 & -0.0330 & 2.9120 \\
$f_{t}^{201306}$ & 128 & -0.0565 & 1.3939 & -1.5636 & 11.541 \\
$f_{t}^{201309}$ & 135 & -0.0247 & 1.5982 & -0.8189 & 7.9047 \\
$f_{t}^{201312}$ & 134 & 0.0359 & 1.3708 & -0.0362 & 4.7350 \\
$f_{t}^{201403}$ & 128 & -0.0006 & 0.9230 & -0.2069 & 3.1277 \\
$f_{t}^{201406}$ & 148 & -0.0079 & 0.8389 & -0.0203 & 3.3904 \\
$f_{t}^{201409}$ & 134 & 0.0137 & 0.7256 & 0.1878 & 4.3808 \\
$f_{t}^{201412}$ & 126 & 0.0474 & 0.7257 & 0.9428 & 9.7890 \\
\hline$R K_{t}^{201203}$ & 46 & 0.6541 & 0.3476 & 1.1704 & 3.9350 \\
$R K_{t}^{201206}$ & 107 & 0.6175 & 0.3727 & 1.7664 & 7.3817 \\
$R K_{t}^{201209}$ & 95 & 0.7239 & 0.3419 & 2.7107 & 15.984 \\
$R K_{t}^{201212}$ & 115 & 0.6482 & 0.3390 & 1.5026 & 6.5683 \\
$R K_{t}^{201303}$ & 105 & 0.7803 & 0.4671 & 1.6055 & 6.7894 \\
$R K_{t}^{201306}$ & 128 & 1.4877 & 2.2604 & 3.5632 & 16.853 \\
$R K_{t}^{201309}$ & 135 & 2.0075 & 2.5408 & 3.6612 & 20.302 \\
$R K_{t}^{201312}$ & 134 & 1.6681 & 1.6150 & 3.0244 & 12.857 \\
$R K_{t}^{201403}$ & 128 & 0.9638 & 0.5082 & 1.3250 & 4.8540 \\
$R K_{t}^{201406}$ & 148 & 0.8350 & 0.5132 & 1.5186 & 6.0937 \\
$R K_{t}^{201409}$ & 134 & 0.5636 & 0.3733 & 1.9800 & 7.7633 \\
$R K_{t}^{201412}$ & 126 & 0.4621 & 0.5464 & 6.7074 & 60.208 \\
\hline
\end{tabular}

Note: The superscripts of $f_{t}$ and $R K_{t}^{f}$ indicate the contract month and year (yyyymm), while $n^{f}$ denotes the number of observations. 
Table 2: Estimation Results for Regression (2)

\begin{tabular}{c|ccccc}
\hline \hline & \multicolumn{3}{|c}{ Explanatory Var. } & & \\
Dep. Var. & Const. & $R_{t}$ & $-T$ & $\bar{R}^{2}$ & Robust $\chi^{2}$ Test \\
\hline$f_{t}^{201203}$ & -0.0644 & 1.0307 & -0.2773 & 0.9161 & 35.377 \\
& $(0.0001)$ & $(0.0001)$ & $(0.0109)$ & & {$[0.0000]$} \\
$f_{t}^{201206}$ & 0.0065 & 0.9906 & 0.1186 & 0.8849 & 2.4506 \\
& $\left(6.48 \times 10^{-5}\right)$ & $\left(4.64 \times 10^{-5}\right)$ & $(0.0007)$ & & {$[0.2937]$} \\
$f_{t}^{201209}$ & -0.0493 & 0.9679 & -0.2907 & 0.9034 & 93.591 \\
& $\left(4.72 \times 10^{-5}\right)$ & $\left(2.38 \times 10^{-5}\right)$ & $(0.0012)$ & & {$[0.0000]$} \\
$f_{t}^{201212}$ & -0.0270 & 0.9659 & -0.0114 & 0.9050 & 110.84 \\
& $\left(4.02 \times 10^{-5}\right)$ & $\left(2.03 \times 10^{-5}\right)$ & $(0.0004)$ & & {$[0.0000]$} \\
$f_{t}^{201303}$ & -0.0469 & 0.8874 & -0.0964 & 0.8737 & 374.42 \\
& $\left(8.77 \times 10^{-5}\right)$ & $\left(3.39 \times 10^{-5}\right)$ & $(0.0011)$ & & {$[0.0000]$} \\
$f_{t}^{201306}$ & -0.1200 & 0.9156 & -0.2494 & 0.8934 & 395.04 \\
& $(0.0002)$ & $\left(2.1413 \times 10^{-5}\right)$ & $(0.0012)$ & & {$[0.0000]$} \\
$f_{t}^{201309}$ & 0.0005 & 0.9750 & 0.1764 & 0.9121 & 29.721 \\
& $\left(8.49 \times 10^{-5}\right)$ & $\left(2.12 \times 10^{-5}\right)$ & $(0.0010)$ & & {$[0.0000]$} \\
$f_{t}^{201312}$ & 0.0326 & 0.9915 & 0.1357 & 0.9272 & 17.566 \\
& $\left(6.13 \times 10^{-5}\right)$ & $\left(3.39 \times 10^{-5}\right)$ & $(0.0010)$ & & {$[0.0002]$} \\
$f_{t}^{201403}$ & 0.0521 & 0.8868 & 0.1558 & 0.8583 & 550.42 \\
& $\left(8.80 \times 10^{-5}\right)$ & $\left(2.38 \times 10^{-5}\right)$ & $(0.0010)$ & & {$[0.0000]$} \\
$f_{t}^{201406}$ & 0.0215 & 0.8777 & 0.03056 & 0.8452 & 681.52 \\
& $\left(3.67 \times 10^{-5}\right)$ & $\left(2.21 \times 10^{-5}\right)$ & $(0.0003)$ & & {$[0.0000]$} \\
$f_{t}^{201409}$ & -0.0370 & 0.9517 & -0.1880 & 0.8644 & 152.35 \\
& $\left(2.23 \times 10^{-5}\right)$ & $\left(2.27 \times 10^{-5}\right)$ & $(0.0005)$ & & {$[0.0000]$} \\
$f_{t}^{201412}$ & 0.0765 & 0.9363 & 0.2092 & 0.9306 & 636.77 \\
& $\left(3.48 \times 10^{-5}\right)$ & $\left(2.84 \times 10^{-5}\right)$ & $(0.0004)$ & & {$[0.0000]$} \\
\hline
\end{tabular}

Note: The superscripts of $f_{t}$ indicate the contract month and year (yyyymm). The robust $\chi^{2}$ test reports the statistics for testing the restrictions $\alpha_{r}=0$ and $\beta_{r}=1$, and follows the $\chi^{2}(2)$ distribution asymptotically, under the null hypothesis. Standard errors via the HAC estimator are given in parentheses, while $P$-values for the robust $\chi^{2}$ test statistic are reported in brackets. 
Table 3: Estimation Results for Regression (3)

\begin{tabular}{|c|c|c|c|c|c|c|}
\hline \multirow[b]{2}{*}{ Dep. Var. } & \multicolumn{4}{|c|}{ Explanatory Var. } & \multirow[b]{2}{*}{$\bar{R}^{2}$} & \multirow[b]{2}{*}{ Robust $\chi^{2}$ Test } \\
\hline & Const. & $R K_{t}$ & $T$ & $T^{2}$ & & \\
\hline \multirow[t]{2}{*}{$R K_{t}^{201203}$} & -0.0271 & 1.6306 & 0.7174 & -0.5520 & 0.9146 & 389.60 \\
\hline & $(0.0001)$ & $(0.0017)$ & $(0.2606)$ & $(5.7535)$ & & {$[0.0000]$} \\
\hline \multirow[t]{2}{*}{$R K_{t}^{201206}$} & -0.0688 & 1.1710 & 1.6999 & -3.6927 & 0.9249 & 92.246 \\
\hline & $(0.0003)$ & $(0.0004)$ & $(0.0118)$ & $(0.0392)$ & & {$[0.0000]$} \\
\hline \multirow{2}{*}{$R K_{t}^{201209}$} & 0.2945 & 1.0652 & 0.4875 & -3.6937 & 0.9362 & 643.32 \\
\hline & $(0.0002)$ & $(0.0004)$ & $(0.0178)$ & $(0.0885)$ & & {$[0.0000]$} \\
\hline \multirow[t]{2}{*}{$R K_{t}^{201212}$} & 0.2627 & 1.2009 & 0.3039 & -2.2745 & 0.9293 & 1305.1 \\
\hline & $(0.0001)$ & $(0.0002)$ & $(0.0071)$ & $(0.0253)$ & & $.0000]$ \\
\hline \multirow[t]{2}{*}{$R K_{t}^{201303}$} & 0.2527 & 1.0340 & 0.9183 & -3.4820 & 0.9378 & 997.40 \\
\hline & $(0.0003)$ & $(0.0005)$ & $(0.0105)$ & $(0.0420)$ & & {$[0.0000]$} \\
\hline \multirow[t]{2}{*}{$R K_{t}^{201306}$} & 0.1276 & 1.0433 & -0.2638 & 0.3662 & 0.9673 & 139.04 \\
\hline & $(0.0017)$ & $\left(8.30 \times 10^{-5}\right)$ & $(0.0766)$ & $(0.1753)$ & & {$[0.0000]$} \\
\hline \multirow[t]{2}{*}{$R K_{t}^{201309}$} & 0.3425 & 1.1760 & -1.4416 & 0.9773 & 0.9548 & 2359.0 \\
\hline & $(0.0002)$ & $(0.0002)$ & $(0.0335)$ & $(0.1246)$ & & {$[0.0000]$} \\
\hline \multirow[t]{2}{*}{$R K_{t}^{201312}$} & 0.2152 & 1.1777 & 0.4865 & -2.1182 & 0.9653 & 1243.3 \\
\hline & $(0.0001)$ & $(0.0002)$ & $(0.0138)$ & $(0.0652)$ & & [0.0000] \\
\hline \multirow[t]{2}{*}{$R K_{t}^{201403}$} & 0.3506 & 0.9952 & -0.9590 & 1.4600 & 0.9341 & 1482.5 \\
\hline & $(0.0002)$ & $(0.0002)$ & $(0.0095)$ & $(0.0433)$ & & {$[0.0000]$} \\
\hline \multirow[t]{2}{*}{$R K_{t}^{201406}$} & 0.2048 & 0.9990 & 0.5594 & -1.6682 & 0.9402 & 1862.7 \\
\hline & $\left(2.53 \times 10^{-5}\right)$ & $(0.0001)$ & $(0.0038)$ & $(0.0110)$ & & {$[0.0000]$} \\
\hline \multirow[t]{2}{*}{$R K_{t}^{201409}$} & 0.1166 & 1.1377 & 0.4779 & -1.5226 & 0.9220 & 781.47 \\
\hline & $\left(5.70 \times 10^{-5}\right)$ & $(0.0004)$ & $(0.0047)$ & $(0.0251)$ & & [0.0000] \\
\hline \multirow{2}{*}{$R K_{t}^{201412}$} & -0.0581 & 1.3572 & 0.7068 & -1.5981 & 0.9684 & 1681.3 \\
\hline & $\left(6.71 \times 10^{-}\right.$ & $\left(8.17 \times 10^{-5}\right)$ & $(0.0024)$ & $(0.0060)$ & & {$[0.0000]$} \\
\hline
\end{tabular}

Note: The superscripts of $R K^{f}$ indicate the contract month and year (yyyymm). The robust $\chi^{2}$ test reports the statistics for testing the restrictions $\alpha_{v}=0$ and $\beta_{v}=1$, and follows the $\chi^{2}(2)$ distribution asymptotically, under the null hypothesis. Standard errors via the HAC estimator are given in parentheses, while $P$-values for the robust $\chi^{2}$ test statistic are reported in brackets.

Table 4: One-Step-Ahead Forecasts for Volatility of Nikkei 225 Returns

(a) RMSFE

\begin{tabular}{c|ccc}
\hline \hline Data & FIEGARCH & RK-HAR-A & RK-ALSV \\
\hline$R K$ & 25.891 & 19.569 & 18.213 \\
\hline
\end{tabular}

(b) Diebold-Mariano Test

\begin{tabular}{cc|cc}
\hline \hline Model 1 & Model 2 & $Z$ & $P$-value \\
\hline FIEGARCH & RK-HAR-A & -1.6890 & 0.0456 \\
FIEGARCH & RK-ALSV & -3.3660 & 0.0004 \\
RK-HAR-A & RK-ALSV & -1.6684 & 0.0476 \\
\hline
\end{tabular}

Note: The test statistic, $Z$, has an asymptotic normal distribution under the null hypothesis. 
Table 5: RMSFE for One-Step-Ahead Forecasts for Volatility of Nikkei 225 Futures

\begin{tabular}{c|cccl}
\hline \hline Data & AR(1)-A & FIEGARCH & RK-HAR-A & RK-ALSV \\
\hline$R K_{t}^{201309}$ & 31.580 & 29.981 & 27.223 & $27.105^{*}$ \\
$R K_{t}^{201312}$ & 21.636 & 23.480 & 23.308 & $17.826^{* *}$ \\
$R K_{t}^{201403}$ & 7.9803 & 8.3802 & 7.4992 & $6.2840^{*}$ \\
$R K_{t}^{201406}$ & 6.1994 & 6.3315 & 6.4050 & 5.1506 \\
$R K_{t}^{201409}$ & 6.8921 & 8.9260 & 4.9410 & 4.0989 \\
$R K_{t}^{201412}$ & 6.0217 & 5.7820 & 5.8207 & $4.9519^{*}$ \\
\hline
\end{tabular}

Note: The superscripts of $R K_{t}^{f}$ indicate the contract month and year (yyyymm). '*', ('**') denotes significant differences between the forecasts of the RK-ALSV model and the second best model at the $5 \%(1 \%)$ significance level.

Table 6: RMSFE for Connected Data of Volatility of Nikkei 225 Futures

\begin{tabular}{c|ccc|crc}
\hline \hline & \multicolumn{3}{|c|}{ Direct Method } & \multicolumn{3}{c}{ Indirect Method } \\
\cline { 2 - 7 } Data & RK $^{f}$-HAR-A & RK $^{f}$-ALSV & AR(1)-A & FIEGARCH & RK-HAR-A & RK-ALSV \\
\hline$R K_{t}^{201309}$ & 10.194 & 12.119 & 11.520 & 17.587 & 12.680 & 8.9877 \\
$R K_{t}^{201312}$ & 4.0814 & 4.3886 & 4.0430 & 4.6122 & 4.7111 & 2.2943 \\
$R K_{t}^{201403}$ & 4.5715 & 4.1080 & 3.6947 & 3.9723 & 3.9539 & 2.0571 \\
$R K_{t}^{201406}$ & 2.9927 & 2.9782 & 2.5067 & 2.6320 & 2.5960 & 1.8416 \\
$R K_{t}^{201409}$ & 3.0065 & 2.8287 & 2.3869 & 2.6344 & 2.3610 & 1.6429 \\
$R K_{t}^{201412}$ & 5.3582 & 4.5186 & 5.5123 & 5.1075 & 5.2661 & 4.6056 \\
\hline
\end{tabular}

Note: The superscripts of $R K_{t}^{f}$ indicate the contract month and year (yyyymm).

Table 7: Utility-Based Measure

\begin{tabular}{|c|c|c|c|c|c|c|c|c|c|c|c|c|c|c|}
\hline \multirow{2}{*}{$\begin{array}{c}\text { Maturity } \\
\text { Date }\end{array}$} & \multicolumn{2}{|c|}{$\overline{\mathrm{AR}(1)-\mathrm{A}}$} & \multicolumn{4}{|c|}{ FIEGARCH } & \multicolumn{4}{|c|}{ RK-HAR-A } & \multicolumn{4}{|c|}{ "RK-ALSV } \\
\hline & $\mu$ & $\bar{\sigma}$ & $\mu$ & $\bar{\sigma}$ & $\Delta_{1}$ & $\Delta_{10}$ & $\mu$ & $\bar{\sigma}$ & $\overline{\Delta_{1}}$ & $\Delta_{10}$ & $\mu$ & $\sigma$ & $\Delta_{1}$ & $\Delta_{10}$ \\
\hline 201309 & -0.6 & 8.0 & 1.1 & 28.0 & 21.3 & 138 & 0.1 & $\overline{1.3}$ & 34.7 & -78.0 & -0.5 & 5.5 & 11.9 & 28.1 \\
\hline 201312 & 0.0 & 1.0 & -0.8 & 79.2 & -227 & -111 & 4.5 & 57.9 & 454 & 571 & 1.7 & 14.7 & 100 & 78.4 \\
\hline 201403 & 0.3 & 4.8 & -0.7 & 14.1 & -66.8 & -85.5 & -0.3 & 8.9 & -23.7 & -47.6 & -0.0 & 4.2 & 2.5 & 19.4 \\
\hline 201406 & 0.2 & 4.0 & -0.0 & 0.9 & 7.1 & 34.1 & -0.0 & 0.9 & 7.2 & 35.3 & -0.1 & 0.9 & 7.1 & 34.4 \\
\hline 201409 & -0.7 & 5.4 & 0.0 & 4.4 & 6.3 & 34.0 & 0.1 & 5.0 & 3.4 & -21.1 & 0.3 & 3.4 & 14.8 & -24.7 \\
\hline 201412 & -0.1 & 0.7 & -0.3 & 6.3 & -16.3 & -43.6 & -0.2 & 2.3 & -2.1 & -11.9 & -0.2 & 2.1 & -1.9 & -10.4 \\
\hline
\end{tabular}

Note: For each model, we report the mean next-day portfolio return $(\mu)$, volatility $(\sigma)$, and the basis point fees $\left(\Delta_{\psi}\right)$ which an investor would pay to switch from the static portfolio for relative risk aversion level, $\psi$. 